\title{
Prediction of difficult airway management in traumatic cervical spine injury: influence of retropharyngeal space extension
}

This article was published in the following Dove Medical Press journal:

Therapeutics and Clinical Risk Management

\author{
Jeongwoo Lee ${ }^{1,2, *}$ \\ Jeong Seob Kim' \\ Sehrin Kang' \\ Yu Seob Shin ${ }^{2,3, *}$ \\ A Ram Doo 1,2
}

'Department of Anesthesiology and Pain Medicine, Chonbuk National University Medical School, Jeonju, South Korea; ${ }^{2}$ Research Institute of Clinical Medicine of Chonbuk National University-Biomedical Research Institute of Chonbuk National University Hospital, Jeonju, South Korea; ${ }^{3}$ Department of Urology, Chonbuk National University Medical School, Jeonju, South Korea

*These authors contributed equally to this work
Correspondence: A Ram Doo Department of Anesthesiology and Pain Medicine, Medical School, Chonbuk National University, 20 Geonji-ro,

Deokjin-gu, Jeonju, Jeollabuk-do 54907, South Korea

Email ruiwin3518@gmail.com
Background: Retropharyngeal hematoma following cervical spine trauma may lead to life-threatening upper airway obstruction and difficult airway management. This retrospective study was performed to investigate whether the extension of retropharyngeal space (RPS) was associated with difficult intubation by direct laryngoscopy in traumatic cervical spine injury.

Patients and methods: Sixty-two patients who had undergone direct endotracheal intubation under general anesthesia for cervical spine surgery were retrospectively identified. Laryngoscopic grade by Cormack-Lehane (C-L) classification was collected; grade 1 or 2 was categorized as easy laryngoscopy, whereas grade 3 or 4 was categorized as difficult laryngoscopy. In these patients, RPS thickness and the proportions of RPS to the vertebral bodies were measured at the 2nd, 5th and 7 th cervical spine levels using magnetic resonance imaging (MRI) of the cervical spine. Measures of RPS were compared between easy and difficult laryngoscopy. Relationships between measures of RPS and difficult laryngoscopy were analyzed with logistic regression analysis.

Results: RPS thickness at C2 was significantly greater in difficult laryngoscopy (median $14.29 \mathrm{~mm}$, IQR: 9.75-18.04) than easy laryngoscopy (median 5.10, IQR: 4.33-5.94, $p<0.001$ ). Proportion of RPS to the $\mathrm{C} 2$ vertebral body were significantly higher in difficult laryngoscopy than in easy laryngoscopy $(p<0.001)$. RPS thickness and the proportion of RPS to the vertebral body were significantly associated with difficult laryngoscopy $(\mathrm{OR}=2.13,95 \% \mathrm{CI}: 1.38-3.30$; $p<0.001$ and $\mathrm{OR}=1.13,95 \% \mathrm{CI}: 1.05-1.21 ; p<0.001$, respectively).

Conclusion: RPS extension at the upper cervical spine level is associated with difficult direct laryngoscopy in traumatic cervical spine injury.

Keywords: cervical spine injury, difficult airway, endotracheal intubation, retropharyngeal space

\section{Introduction}

Airway management is a major concern in patients with traumatic cervical spine injury. Although high-level cervical spine injury is associated with severe respiratory compromise requiring aggressive airway management, such management is difficult. Manual in-line stabilization (MILS) is routinely used to immobilize the cervical spine during endotracheal intubation, because excessive cervical motion may deteriorate spinal cord injury by mechanical cord compression or secondary ischemia. However, limited head extension during this technique increases the difficulty of aligning the oropharyngeal and laryngeal axes and may disturb the epiglottis and vocal cord visibility in direct laryngoscopy, resulting in difficult laryngoscopy and endotracheal intubation.

Importantly, retropharyngeal hemorrhage caused by cervical spine fracture, whiplash injury, vessel injury of the neck or spontaneously is reportedly associated with respiratory compromise. ${ }^{1-6} \mathrm{~A}$ few case reports have revealed that severe 
retropharyngeal hematoma following traumatic cervical spine injury can lead to life-threatening respiratory complications, such as upper airway obstruction and resultant difficult endotracheal intubation. ${ }^{1,2,7}$ The retropharyngeal space (RPS) is a potential space that extends from the base of the skull to the superior mediastinum at the level of the second thoracic vertebra; it lies posterior to buccopharyngeal fascia and pharynx and anterior to the prevertebral fascia and musculature. ${ }^{8}$ Theoretically, a large retropharyngeal hematoma may extend anteriorly and may displace and compress the pharyngeal cavity, resulting in difficult airway management. However, no report has clearly elucidated the relationship between the severity of RPS extension and the difficulty of endotracheal intubation in patients with traumatic cervical spine injury. The aim of this retrospective study was to investigate whether radiologic measurement of RPS may be a useful predictor of difficult endotracheal intubation by direct laryngoscopy in patients with traumatic cervical spine injury.

\section{Patients and methods}

\section{Patients}

This retrospective study was approved by the institutional review board of Chonbuk National University Hospital, which waived the requirement for informed consent. The study was conducted in accordance with the Good Clinical Practice and the International Conference on Harmonization guidelines and in conformity with the ethical principles of the Declaration of Helsinki. The medical records of 250 patients who underwent surgery under general anesthesia to treat traumatic cervical spine injury, at a single institution during the period from January 2011 to December 2018, were evaluated. Among them, 79 patients who had undergone endotracheal intubation by direct laryngoscopy with MILS to secure the airway during anesthesia induction were identified, based on anesthesia records in the electronic medical records. Demographic data, cause of cervical spine injury, and diagnosis by preoperative magnetic resonance imaging (MRI) were also recorded. Seventeen patients who had undergone delayed surgery (beyond 72 hours after obtaining MRI scan of the cervical spine) were excluded from the analysis to clarify the study results.

Anesthetists reported grading of the direct laryngoscopic view using the Cormack-Lehane (C-L) classification system, and any other descriptions of airway management or complication, either during anesthesia or in the postanesthesia care unit, were retrospectively collected. C-L grade 1 or 2 was categorized as easy laryngoscopy; grade 3 or 4 was categorized as difficult laryngoscopy.

\section{Quantitative analysis of RPS in MRI}

To obtain the RPS thickness, we measured the maximal anteroposterior distance between the posterior margin of the pharynx, larynx or trachea and the anterior bony margin of cervical vertebra in the median sagittal plane of the T2-weighted MR sequences of cervical spine, which were scanned within 72 hours prior to surgery. The proportion of RPS to vertebral body was calculated as a ratio of the RPS thickness to antero-posterior (A-P) diameter of each vertebral body multiplied by 100 . The measurements were performed in the same manner in the upper (2nd), middle (5th) and lower ( 7 th) cervical vertebra (described in Figure 1). We also measured the A-P distances of the patient's airway including oropharynx, hypopharynx, larynx and trachea. The landmarks for the measurements are as follows: oropharynx, from the root of tongue to the posterior pharyngeal wall; hypopharynx, from the vallecular fossa to the posterior pharyngeal wall; larynx, from anterior commissure to posterior commissure in the true vocal cord; and trachea, from anterior to posterior trachea wall, $5 \mathrm{~cm}$ above the carina.

All measurements were analyzed by three anesthesiologists who were blinded to anesthesia records, including laryngoscopic grade; a consensus was reached for each measurement. In cases of disagreement with a difference of more than $10 \%$,

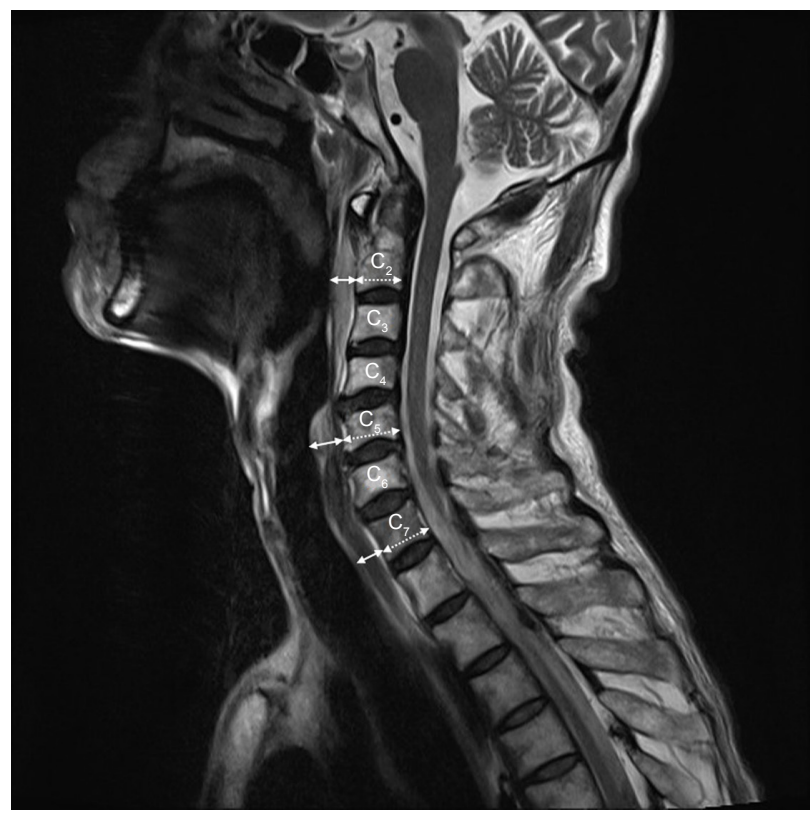

Figure I Measurements of RPS in 2nd, 5th and 7th cervical spine level in median sagittal MRI images.

Notes: The thickness of RPS (solid double arrow) is measured by the anteroposterior distance between the posterior margin of pharynx, larynx or trachea and the anterior bony margin of cervical vertebra. The proportion of RPS to vertebral body was calculated as a ratio of the thickness of RPS to A-P diameter of each vertebral body (dotted double arrow) multiplied by 100.

Abbreviations: A-P, antero-posterior; MRI, magnetic resonance imaging; RPS, retropharyngeal space. 
the images were simultaneously reviewed by all three anesthesiologists and discussed until a consensus was reached.

\section{Statistics}

All statistical analyses were performed using Sigma Plot version 12.5. (Systat Software Inc., San Jose, CA, USA). All descriptive statistics are expressed as mean $\pm \mathrm{SD}$, median (25th-75th percentile), percentage or the number of patients. Continuous variables including patient demographics were analyzed by Student's $t$-test or Mann-Whitney rank-sum test after normality test. Nonparametric variables were analyzed by the Mann-Whitney rank-sum test.

First, we compared the radiologic measures of RPS between easy and difficult laryngoscopy group using Student's $t$-test or Mann-Whitney rank-sum test. Second, we investigated the correlation between the radiologic measures of RPS and difficult laryngoscopy. The radiologic measures associated with difficult laryngoscopy were integrated into a logistic regression model, and the ORs and their 95\% CIs were calculated. The predictive power of the regression model was calculated using a receiver operating characteristic (ROC) curve and the area under the curve (AUC). After a logistic regression model was developed, further investigation included Spearman's Rank Correlation analysis to verify the relationships between radiologic measures of RPS and the C-L grade. A $p$-value of $<0.05$ was considered to be statistically significant.

\section{Results}

\section{Patient characteristics and quantitative measurement of RPS}

We analyzed 62 patients in this study, 49 with easy (34 of C-L grade 1 and 15 of grade 2) and 13 with difficult laryngoscopy (12 of C-L grade 3 and 1 of grade 4). Patient characteristics are described in Table 1. The median thickness of RPS at the 2nd cervical spine level was $14.29 \mathrm{~mm}$ (IQR 9.75-18.04) in the difficult laryngoscopy group, which was significantly higher than that in the easy laryngoscopy group (median 5.10, IQR: 4.33-5.94, $p<0.001$ ) (Table 2). However, RPS thickness at both C5 and C7 was not significantly different between easy and difficult laryngoscopy groups ( $p=0.067$ and $p=0.142$, respectively). The proportion of RPS thickness to A-P diameter of the 2nd cervical vertebral body was also significantly higher in the difficult laryngoscopy group, compared with the easy laryngoscopy group $(p<0.001)$. The A-P diameters of the patients' airways were also evaluated. The difficult laryngoscopy group exhibited a smaller A-P diameter of oropharynx, hypopharynx and larynx, compared with the easy laryngoscopy group $(p=0.005, p=0.002$ and $p=<0.001$, respectively) (Table 2 ).

\section{Prediction of difficult laryngoscopy in patients with traumatic cervical spine injury}

Univariate analysis revealed that difficult laryngoscopy during anesthesia induction in cervical spine injury patient was significantly associated with RPS thickness at C2, the proportion of RPS to the 2 nd cervical vertebral body and the A-P diameter of the patient's airway in median sagittal MRI images (ORs and 95\% CIs are shown in Table 3).

We created an ROC curve $(\mathrm{AUC}=0.97,95 \% \mathrm{CI}$ : $0.94-1.01 ; p<0.001)$ to establish the cutoff point of the RPS predictive of difficult laryngoscopy in patients with cervical spine injury, especially with enlargement of the RPS. Cutoff values of RPS thickness and the proportion of RPS to the 2 nd

Table I Characteristics of patients

\begin{tabular}{|c|c|c|c|}
\hline & Easy laryngoscopy $(n=49)$ & Difficult laryngoscopy $(n=\mid 3)$ & $P$-value \\
\hline Gender (M/F) & $40 / 9$ & $11 / 2$ & 1.000 \\
\hline Age (years) & $54.9 \pm 16.2$ & $55.5 \pm 15.4$ & 0.914 \\
\hline Height $(\mathrm{cm})$ & $167.4 \pm 8.1$ & $168.3 \pm 5.6$ & 0.693 \\
\hline Weight (kg) & $64.5 \pm 10.6$ & $65.4 \pm 11.7$ & 0.789 \\
\hline BMI $\left(\mathrm{kg} / \mathrm{m}^{2}\right)$ & $22.9 \pm 2.7$ & $23.0 \pm 3.2$ & 0.970 \\
\hline ASA PS (I/II/III//IV) & $15 / 25 / 8 / 1 / 0$ & $0 / 7 / 5 / 0 / 1$ & 0.241 \\
\hline Cause of cervical injury & & & 0.287 \\
\hline Fall down & 8 & 7 & \\
\hline Slip down & 8 & 1 & \\
\hline Car accident & 29 & 4 & \\
\hline Pedestrian accident & 4 & 1 & \\
\hline Time elapsed from MRI to surgery (hours) & $22.0(12.5-42.0)$ & $14.0(6.0-39.0)$ & 0.358 \\
\hline
\end{tabular}

Note: Values are expressed as numbers, mean \pm SD or median (25th-75th percentile).

Abbreviations: ASA PS, American Society of Anesthesiologist physical status; BMI, body mass index; F, female; M, male; MRI, magnetic resonance imaging. 
Table 2 Quantitative measurement of retropharyngeal space between easy and difficult laryngoscopy during anesthesia induction in patients with traumatic cervical spine injury

\begin{tabular}{|c|c|c|c|}
\hline & $\begin{array}{l}\text { Easy } \\
\text { laryngoscopy }\end{array}$ & $\begin{array}{l}\text { Difficult } \\
\text { laryngoscopy }\end{array}$ & $P$-value \\
\hline \multicolumn{4}{|c|}{ MRI findings of cervical spine } \\
\hline \multicolumn{4}{|c|}{ Thickness of RPS $(\mathrm{mm})^{\dagger}$ at } \\
\hline $\mathrm{C} 2$ & $5.10(4.33-5.94)$ & $14.29(9.75-18.04)$ & $<0.00 I^{*}$ \\
\hline C5 & $13.65 \pm 2.45$ & $15.43 \pm 3.52$ & 0.067 \\
\hline $\mathrm{C7}$ & $13.07 \pm 3.17$ & $14.93 \pm 4.77$ & 0.142 \\
\hline \multicolumn{4}{|c|}{ Proportion of RPS to vertebral body (\%) ${ }^{\dagger \dagger}$ at } \\
\hline $\mathrm{C} 2$ & $29.4(25.0-35.8)$ & $79.0(54.5-110.4)$ & $<0.001 *$ \\
\hline C5 & $80.2 \pm 14.2$ & $86.4 \pm 24.9$ & 0.305 \\
\hline $\mathrm{C7}$ & $72.7 \pm 15.9$ & $78.1 \pm 23.0$ & 0.384 \\
\hline \multicolumn{4}{|c|}{ Anteroposterior diameter of airway $(\mathrm{mm})$} \\
\hline Oropharynx & $13.5 \pm 4.1$ & $7.9 \pm 3.0$ & $0.005^{* *}$ \\
\hline Hypopharynx & $19.3 \pm 3.6$ & $13.6 \pm 4.1$ & $0.002^{* *}$ \\
\hline Larynx & $19.4 \pm 3.2$ & $13.7 \pm 4.6$ & $<0.001 * *$ \\
\hline Trachea & $14.1 \pm 2.3$ & $14.5 \pm 2.3$ & 0.706 \\
\hline
\end{tabular}

Notes: ${ }^{\dagger}$ Anteroposterior distance between the posterior margin of pharynx or trachea and the anterior bony margin of cervical vertebra at the $2 \mathrm{nd}$, 5th and 7 th cervical levels, which were measured on median sagittal MRI images. ${ }^{\dagger+}$ Calculated as a ratio of RPS to A-P diameter of each vertebral body multiplied by 100 . $* P<0.05$ by the Mann-Whitney rank-sum test between easy and difficult laryngoscopy groups. $* * P<0.05$ by Student's $t$-test between easy and difficult laryngoscopy groups.

Abbreviations: A-P, antero-posterior; MRI, magnetic resonance imaging; RPS, retropharyngeal space.

cervical vertebral body were $7.94 \mathrm{~mm}$ and 48.4\% $(p<0.001$ and $p<0.001$, respectively) (Figure 2).

\section{Association between laryngoscopic grade and RPS thickness}

In linear regression analysis, there was a linear correlation between C-L grade and RPS thickness at C2 ( $\mathrm{r}=0.738$, $\left.\mathrm{r}^{2}=0.545 ; p<0.001\right)$, as well as between $\mathrm{C}$-L grade and proportion of RPS to the 2 nd cervical vertebral body ( $r=0.710$,

Table 3 Univariate logistic regression analysis for the prediction of difficult laryngoscopy in patients with traumatic cervical spine injury

\begin{tabular}{|l|l|l|l|}
\hline & OR & $\mathbf{9 5 \%} \mathbf{C l}$ & P-value \\
\hline Thickness of RPS at C2 (mm) & 2.13 & $1.38-3.30$ & $<0.00 I^{*}$ \\
\hline $\begin{array}{l}\text { Proportion of RPS to vertebral } \\
\text { body at C2 (\%) }\end{array}$ & 1.13 & $1.05-1.21$ & $<0.00 I^{*}$ \\
\hline $\begin{array}{l}\text { Anteroposterior diameter } \\
\text { of airway (mm) }\end{array}$ & & & \\
\hline Oropharynx & 0.59 & $0.38-0.91$ & $0.016^{*}$ \\
\hline Hypopharynx & 0.61 & $0.40-0.92$ & $0.020^{*}$ \\
\hline Larynx & 0.61 & $0.41-0.93$ & $0.02 I^{*}$ \\
\hline
\end{tabular}

Note: $* P<0.05$ was considered statistically significant.

Abbreviation: RPS, retropharyngeal space.

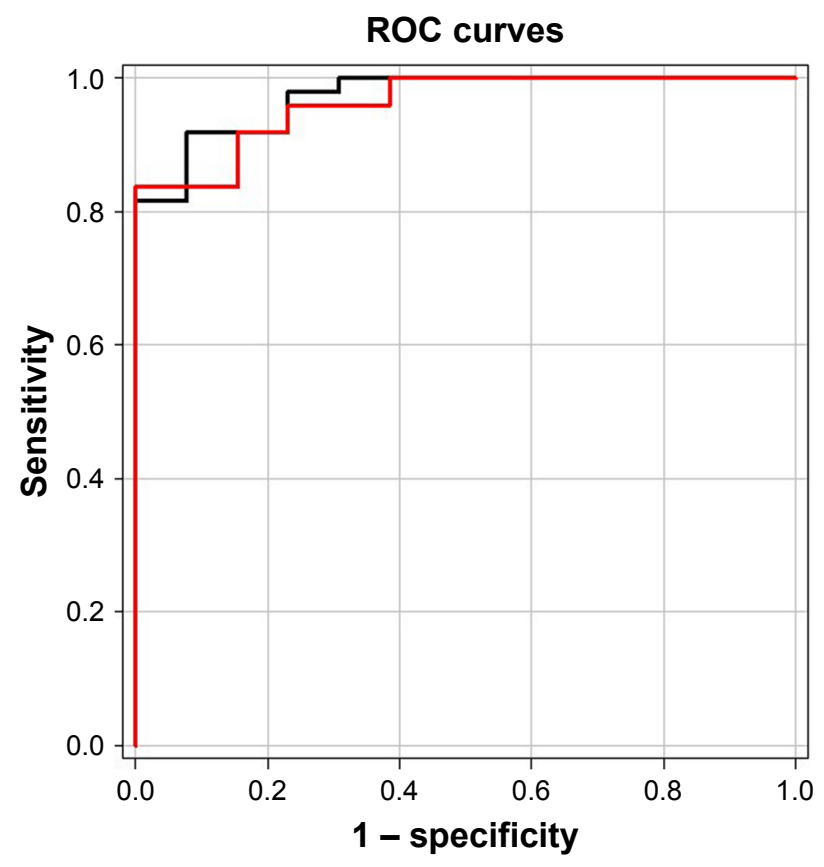

- Thickness of RPS at 2nd cervical spine, AUC $=0.97$ - Proportion of RPS to 2nd cervical vertebra, AUC $=0.96$

Figure 2 ROC curve and AUC of the RPS at the 2nd cervical spine level. Notes: The areas under the receiver operator characteristic curve are 0.97 (95\% Cl: 0.94-I.0I; $p<0.00 \mathrm{I}$ ) and 0.96 (95\% Cl: 0.92-1.0I; $p<0.00 \mathrm{I})$, respectively. Cutoff values of RPS thickness and the proportion of RPS to the 2 nd cervical vertebral body are $7.94 \mathrm{~mm}$ and $48.4 \%(p<0.00 \mathrm{I}$ and $p<0.00 \mathrm{I}$, respectively).

Abbreviations: AUC, area under the curve; ROC, receiver operating characteristic; RPS, retropharyngeal space.

$\mathrm{r}^{2}=0.505 ; p<0.001$ ) (Figure 3). However, A-P diameters of airway, including oropharynx, hypopharynx and larynx, were not significantly correlated with laryngoscopic grade.

\section{Discussion}

This study demonstrated that the extension of RPS at the upper cervical spine level was associated with difficult laryngoscopy and endotracheal intubation in patients with traumatic cervical spine injury. Several authors have reported that large retropharyngeal hematoma may cause life-threatening upper airway obstruction and respiratory distress. ${ }^{1-4,7}$ However, there has been no quantitative assessment of the ability of RPS to predict difficult airway management. The authors hypothesized that the severity of RPS extension would be related to the difficulty of endotracheal intubation by direct laryngoscopy in patients with traumatic cervical spine injury. As our study showed, RPS thickness or the proportion of RPS to the 2nd cervical vertebral body was linearly correlated with laryngoscopic grade. Therefore, extension of RPS caused by swelling or hemorrhage might be the warning sign of the possibility of difficult airway 
A

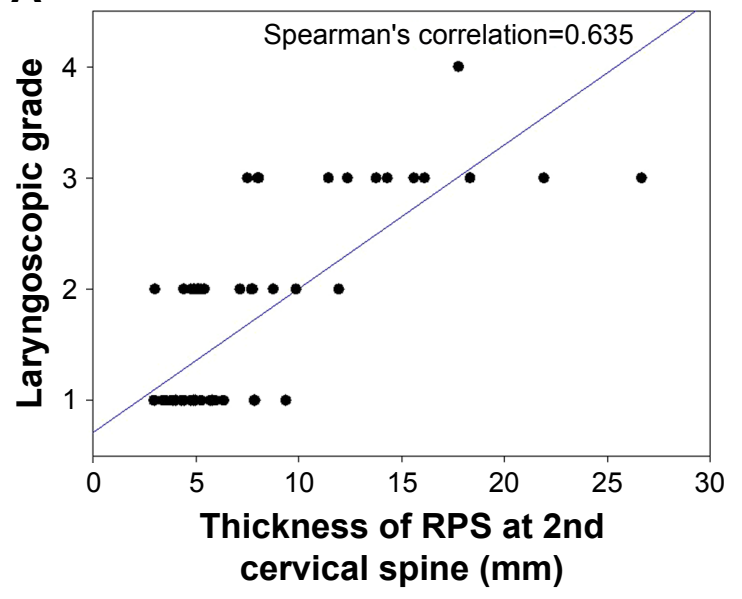

B

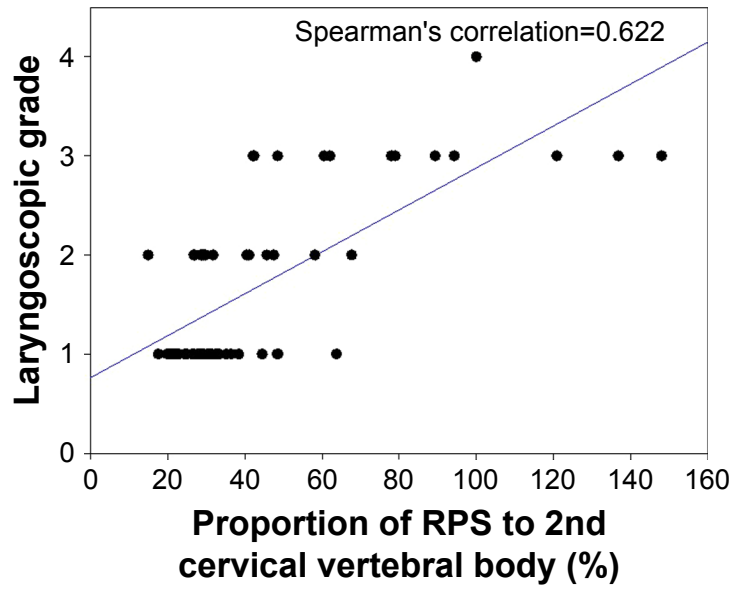

Figure 3 The relationships between laryngoscopic grade using Cormack-Lehane classification and (A) RPS thickness at the 2nd cervical spine level, and (B) proportion of RPS to A-P diameter of the 2 nd cervical vertebral body.

Abbreviations: A-P, antero-posterior; RPS, retropharyngeal space.

management in cervical spine trauma. The authors suggest that careful planning and preparation for predicted difficult airway management should be performed if RPS thickness at $\mathrm{C} 2$ exceeds $7.94 \mathrm{~mm}$ or if the proportion of RPS to the 2nd cervical vertebral body exceeds $48.4 \%$ in MRI images scanned prior to surgery.

The results of this study may be explained partly by the curvature of the Macintosh laryngoscope blade used routinely in our institution. All direct laryngoscopy was performed using a No. 3 classic Macintosh laryngoscope blade $^{\circledR}$ (Heine Optotechnik, Herrsching, Germany) in this study. The Macintosh laryngoscope blade is a commonly used curved-type blade, which is wider than the straight-type blade. The curved laryngoscope blade must be inserted with curvature between the tongue and posterior pharyngeal wall, and the tip of the blade should be placed into the vallecular fossa, which is the angle created by the epiglottis and base of the tongue, for oro-tracheal intubation. Forward and upward movement of the laryngoscope handle leads the epiglottis to be drawn upwards exposing the laryngeal structure. However, RPS extension at the upper cervical spine level may disturb laryngeal exposure by preventing passage of the laryngoscope blade through the narrow oropharyngeal cavity. Moreover, a protruded posterior pharyngeal wall may cause an unexpectedly poor laryngeal view during direct laryngoscopy.

The maximum normal thicknesses of the retropharyngeal and prevertebral space on the lateral plain radiography of neck have been reported to be $8-11 \mathrm{~mm}$ at $\mathrm{C} 1,6 \mathrm{~mm}$ at $\mathrm{C} 2,7 \mathrm{~mm}$ at $\mathrm{C} 3,8 \mathrm{~mm}$ at $\mathrm{C} 4,22 \mathrm{~mm}$ at $\mathrm{C} 5,19-20 \mathrm{~mm}$ at $\mathrm{C} 6$ and $20-21 \mathrm{~mm}$ at $\mathrm{C} 7 .{ }^{9}$ Meanwhile, the thickness of the RPS varies widely in adults, so that these absolute measurements may be of limited use. Some investigators have established a method to evaluate RPS thickness by measuring the ratio of RPS to the A-P diameter of the cervical vertebral body. ${ }^{10}$ In this study, RPS thickness at $\mathrm{C} 2$ and the proportion of RPS to the 2 nd cervical vertebral body were significant variables in a univariate logistic regression model, and therefore, both variables may be valuable predictors of difficult airway in patients with traumatic cervical spine injury. Although several radiologic predictors for difficult laryngoscopy have been suggested using plain radiography, ${ }^{11-14}$ these studies recruited a small number of patients; furthermore, their variables are complicated to measure and apply in clinical practice. In contrast, with pre-scanned MRI images of the cervical spine, physicians may easily gain valuable information for airway management such as RPS thickness and the proportion of RPS to the vertebral body, as described in Figure 1. Careful preoperative preparation for difficult airway management should be planned if extension of RPS was detected in pre-scanned MRI of the cervical spine.

Recently, new intubation equipment, including various types of video laryngoscopes, has been developed and introduced for the management of difficult airway. Video laryngoscopes have been reported to be more useful in managing difficult airway than standard direct laryngoscopy; however, several studies have revealed that standard direct laryngoscopy can be efficaciously and safely used with MILS techniques, even in cases of cervical spine injury. ${ }^{15-21}$ However, most prospective studies have limitations in that they simulated difficult airway scenarios solely by using MILS. The effects of RPS extension on airway management 
using other airway equipment, such as video laryngoscopy, should be investigated in future studies.

There are a few study limitations. First, several clinical predictors of difficult laryngoscopy, such as Mallampati classification, thyromental distance (TMD) and mandibular retrognathia, can be useful to predict difficult laryngoscopy and intubation. ${ }^{22,23}$ However, these individual physical parameters were not evaluated due to the limitation of the retrospective study. Second, the severity of RPS extension cannot be precisely matched between the time of MRI scanning and endotracheal intubation under general anesthesia. To minimize this discrepancy, we excluded patients who had undergone delayed surgery (beyond 72 hours after MRI scanning). Third, the structure of the cervical spine during general anesthesia might be different from that in the awake state due to the loss of consciousness and the usage of neuromuscular blocking agent. However, the MILS technique was used to neutrally stabilize the cervical spine and minimize spinal cord injury in all patients. Fourth, as the current study in not randomized, the potential confounders that may affect the results may exist.

\section{Conclusion}

RPS extension at the upper cervical spine level may be associated with difficult direct laryngoscopy and endotracheal intubation in patients with traumatic cervical spine injury. Airway management plan in patients with traumatic cervical spine injury should include careful evaluation of RPS.

\section{Acknowledgment}

This study was supported by funds from the Biomedical Research Institute of Chonbuk National University Hospital, Jeonju, South Korea.

\section{Author contributions}

All authors contributed toward data analysis, drafting and critically revising the paper, gave final approval of the version to be published and agree to be accountable for all aspects of the work.

\section{Disclosure}

The authors report no conflicts of interest in this work.

\section{References}

1. Matthews S, Shenvi CL. Airway obstruction and neurogenic shock due to severe cervical spine injury. Am J Emerg Med. 2017;35(1): 196.e1-196. e2. doi:10.1016/j.ajem.2016.06.108
2. Nurata H, Yilmaz MB, Borcek AO, Oner AY, Baykaner MK. Retropharyngeal hematoma secondary to whiplash injury in childhood: a case report. Turk Neurosurg. 2012;22(4):521-523. doi:10.5137/1019-5149. JTN.4011-10.0

3. Kudo S, Fukushima K, Hashimoto M, Furutake M, Tanaka K, Okada K. Airway and circulatory collapse due to retropharyngeal hematoma after blunt vertebral artery injury. Am J Emerg Med. 2017;35(5):806.e5-806. e7. doi:10.1016/j.ajem.2016.06.095

4. Calogero CG, Miller AC, Greenberg MR. Life-threatening retropharyngeal hemorrhage secondary to rupture of the inferior thyroid artery. Case Rep Emerg Med. 2015;2015:789076.

5. Hirota K, Hirata K, Shibata S, Shigematsu K, Higa K, Yamaura K. Risk vessels of retropharyngeal hematoma during stellate ganglion block. Reg Anesth Pain Med. 2017;42(6):778-781. doi:10.1097/AAP. 0000000000000644

6. Ditkofsky N, Hanna T. Images in clinical medicine. Spontaneous retropharyngeal hematoma. $N$ Engl J Med. 2016;374(3):e3. doi:10.1056/ NEJMicm1505596

7. Lazott LW, Ponzo JA, Puana RB, Artz KS, Ciceri DP, Culp WC Jr. Severe upper airway obstruction due to delayed retropharyngeal hematoma formation following blunt cervical trauma. BMC Anesthesiol. 2007;7:2. doi:10.1186/1471-2253-7-2

8. Debnam JM, Guha-Thakurta N. Retropharyngeal and prevertebral spaces: anatomic imaging and diagnosis. Otolaryngol Clin North Am. 2012;45(6):1293-1310. doi:10.1016/j.otc.2012.08.004

9. Penning L. Prevertebral hematoma in cervical spine injury: incidence and etiologic significance. AJR Am J Roentgenol. 1981;136(3):553-561. doi:10.2214/ajr.136.3.553

10. Chong VF, Fan YF. Radiology of the retropharyngeal space. Clin Radiol. 2000;55(10):740-748. doi:10.1053/crad.2000.0510

11. Kamalipour H, Bagheri M, Kamali K, Taleie A, Yarmohammadi H. Lateral neck radiography for prediction of difficult orotracheal intubation. Eur J Anaesthesiol. 2005;22(9):689-693.

12. Chou HC, Wu TL. Mandibulohyoid distance in difficult laryngoscopy. Br J Anaesth. 1993;71(3):335-339.

13. Khan ZH, Arbabi S. Diagnostic value of the upper lip bite test in predicting difficulty in intubation with head and neck landmarks obtained from lateral neck X-ray. Indian J Anaesth. 2013;57(4):381-386. doi:10.4103/0019-5049.118567

14. White A, Kander PL. Anatomical factors in difficult direct laryngoscopy. Br J Anaesth. 1975;47(4):468-474.

15. Hindman BJ, Fontes RB, From RP, et al. Intubation biomechanics: laryngoscope force and cervical spine motion during intubation in cadaverseffect of severe distractive-flexion injury on C3-4 motion. J Neurosurg Spine. 2016;25(5):545-555. doi:10.3171/2016.3.SPINE1640

16. Hindman BJ, From RP, Fontes RB, et al. Intubation biomechanics: laryngoscope force and cervical spine motion during intubation in cadaverscadavers versus patients, the effect of repeated intubations, and the effect of Type II odontoid fracture on C1-C2 motion. Anesthesiology. 2015; 123(5):1042-1058. doi:10.1097/ALN.0000000000000830

17. Holmes MG, Dagal A, Feinstein BA, Joffe AM. Airway management practice in adults with an unstable cervical spine: the harborview medical center experience. Anesth Analg. 2018;127(2):450-454. doi:10.1213/ANE.0000000000003374

18. Ko JI, Ha SO, Koo MS, et al. Comparison of intubation times using a manikin with an immobilized cervical spine: Macintosh laryngoscope vs. GlideScope vs. fiberoptic bronchoscope. Clin Exp Emerg Med. 2015;2(4):244-249. doi:10.15441/ceem.15.043

19. Sulser S, Ubmann D, Schlaepfer M, et al. C-MAC videolaryngoscope compared with direct laryngoscopy for rapid sequence intubation in an emergency department: a randomised clinical trial. Eur J Anaesthesiol. 2016;33(12):943-948. doi:10.1097/EJA.0000000000000525

20. Vijayakumar V, Rao S, Shetty N. A comparison of Macintosh and Airtraq Laryngoscopes for endotracheal intubation in adult patients with cervical spine immobilization using manual in line axial stabilization: a prospective randomized study. J Neurosurg Anesthesiol. 2016;28(4): 296-302. doi:10.1097/ANA.0000000000000224 
21. Yoo JY, Park SY, Kim JY, Kim M, Haam SJ, Kim DH. Comparison of the McGrath videolaryngoscope and the Macintosh laryngoscope for double lumen endobronchial tube intubation in patients with manual inline stabilization: A randomized controlled trial. Medicine (Baltimore). 2018;97(10):e0081. doi:10.1097/MD.0000000000010081

22. Kheterpal S, Healy D, Aziz MF, et al. Incidence, predictors, and outcome of difficult mask ventilation combined with difficult laryngoscopy: a report from the multicenter perioperative outcomes group. Anesthesiology. 2013;119(6):1360-1369. doi:10.1097/ALN.0000435832.39353.20
23. Munster T, Hoffmann M, Schlaffer S, Ihmsen H, Schmitt H, Tzabazis A. Anatomical location of the vocal cords in relation to cervical vertebrae: A new predictor of difficult laryngoscopy? Eur J Anaesthesiol. 2016 33(4):257-262. doi:10.1097/EJA.0000000000000430

\section{Publish your work in this journal}

Therapeutics and Clinical Risk Management is an international, peerreviewed journal of clinical therapeutics and risk management, focusing on concise rapid reporting of clinical studies in all therapeutic areas outcomes, safety, and programs for the effective, safe, and sustained use of medicines. This journal is indexed on PubMed Central, CAS
EMBase, Scopus and the Elsevier Bibliographic databases. The manuscript management system is completely online and includes a very quick and fair peer-review system, which is all easy to use. Visit http://www.dovepress.com/testimonials.php to read real quotes from published authors.

Submit your manuscript here: http://www.dovepress.com/therapeutics-and-clinical-risk-management-journal 